Annals of Pure and Applied Mathematics

Vol. 14, No. 1, 2017, 69-75

ISSN: 2279-087X (P), 2279-0888(online)

Published on 7 July 2017

www.researchmathsci.org

DOI: http://dx.doi.org/10.22457/apam.v14n1a9

Annals of

Pure and Applied Mathematics

\title{
Wirelength of Circulant Networks into Wheel Related Graphs
}

\author{
Jasintha Quadras and S. Sarah Surya* \\ Department of Mathematics, Stella Maris College \\ Chennai 600 086, Tamilnadu, India. \\ *Corresponding author. Email: sara24solomon@gmail.com
}

Received 10 May 2017; accepted 22 June 2017

\begin{abstract}
Circulant graphs are an important class of topological structures of interconnection networks which have been used for decades in the design of computer and telecommunication networks due to their optimal fault tolerance and routing capabilities. In this paper, we consider the problem of embedding the circulant networks into gear and helm graphs to minimize the wirelength.
\end{abstract}

Keywords: embedding, wirelength, edge congestion, circulant, gear graph, helm graph

AMS Mathematics Subject Classification (2010): $05 \mathrm{C} 78$

\section{Introduction}

The geometrical structure of any communication system including internet is based on graph. The logical setup of a computer is designed with the help of graph [13]. An interconnection network can be modelled as a graph. It consists of hardware and software entities that are interconnected to facilitate efficient computation and communication. These entities can be in the form of processors, processes, memory modules or computer systems. In other words, an interconnection network of a system provides logically a specific way in which all components of the system are connected. In this, the simulation of one architecture by another is important. The problem of simulating one network by another is modelled as a graph embedding problem. The need for efficient embedding stems from at least two different directions. If a network A can be embedded in a network $\mathrm{B}$, then all the algorithms developed for parallel processing with network A can be easily transported onto another processor network B. Secondly, mapping parallel algorithms onto parallel architectures often leads to embedding of the control or data flow graphs of the algorithms into the underlying graph of the network. While the general problem of graph embedding is difficult, by exploiting the special structure of the interconnection schemes, a number of results relating to optimal embedding of one class of networks into another have been developed. Embedding the structures may result in substantial savings in communication time. The transmission delay is an important measure for the global communication efficiency of an interconnection network.

Circulant graphs are an important class of topological structures of interconnection networks which have been used for decades in the design of computer and 


\section{Jasintha Quadras and S. Sarah Surya}

telecommunication networks due to their optimal fault tolerance and routing capabilities. They also constitute the basis for designing certain data alignment networks for complex memory systems. Undirected circulant networks arise in the context of Mesh Connected Computer suited for parallel processing of data, such as the well-known ILLIAC type computers. By using circulant graph, we can adapt the performance of the network to the user needs.

The quality of an embedding can be measured by certain cost criteria, namely dilation, expansion, congestion and wirelength. The dilation of an embedding is the maximum distance between the images of adjacent nodes. It is the measure for the communication time needed when simulating one network on another. The bandwidth is the dilation if the host graph is a path. The expansion of an embedding $f$ is the ratio of the number of vertices of $H$ to the number of vertices of $G$.

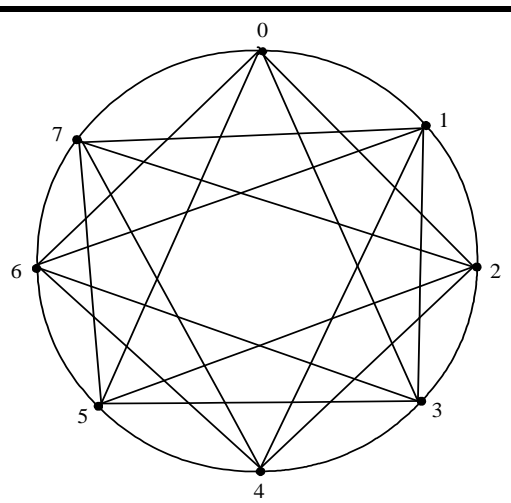

Figure 1: Circulant graph $G(8 ;\{1,2,3\})$

The congestion of an embedding $f$ of $G$ into $H$ is the maximum number of edges of the graph $G$ that are embedded on any single edge of $H$ and the wirelength is nothing but the congestion sum. The problem of embedding is NP-complete[6].

There are several results on the congestion problem of various architectures such as complete trees in hypercubes [1], hypercubes into grids [2], ladders and caterpillars into hypercubes [3], binary trees into hypercubes [4], complete binary trees into grids and extended grids with total vertex congestion 1 [12], incomplete hypercube in books [5], $\mathrm{m}$ sequencial $k$-ary trees into hypercubes [15], ternary tree into hypercube [7], enhanced and augmented hypercube into complete binary tree [9], embeddings of circulant networks [14] and hypercubes into cylinders, snakes and caterpillars [10] .

In this paper, we consider the problem of embedding the circulant networks into gear and helm graphs to minimize the wirelength.

\section{Basic concepts}

In this section, we discuss the preliminaries required for this paper.

Definition 2.1. [2] Let $G$ and $H$ be finite graphs with $n$ vertices. $V(G)$ and $V(H)$ denote the vertex sets of $G$ and $H$ respectively. $E(G)$ and $E(H)$ denote the edge sets of $G$ and $H$ respectively. An embedding $f$ of $G$ into $H$ is defined as follows:

(i) fis a injective map from $V(G) \rightarrow V(H)$ 
Wirelength of Circulant Networks into Wheel Related Graphs

(ii) $P_{f}$ is an injective map from $E(G)$ to $\left\{P_{f}(f(u), f(v)): P_{f}(f(u), f(v))\right.$ is a path in $H$ between $f(u)$ and $f(v)$ \}. The graph $G$ that is being embedded is called a virtual graph or a guest graph and $H$ is called a host graph. Some authors use the name labeling instead of embedding.

Definition 2.2. [2] The edge congestion of an embedding $f$ of $G$ into $H$ is the maximum number of edges of the graph $G$ that are embedded on any single edge of $H$. Let $E C_{f}(G$, $H(e))$ denote the number of edges $(u, v)$ of $G$ such that $e$ is in the path $P_{f}(u, v)$ between $f$ $(u)$ and $f(v)$ in $H$. In other words,

$$
E C_{f}(G, H(e))=\left|\left\{(u, v) \in E(G): e \in P_{f}(u, v)\right\}\right|
$$

where $P_{f}(u, v)$ denotes the path between $f(u)$ and $f(v)$ in $H$ with respect to $f$.

The edge congestion problem of a graph $G$ into $H$ is to find an embedding of $G$ into $H$ that induces $E C(G, H)$.

Definition 2.3. [11] The wirelength of an embedding $f$ of $G$ into $H$ is given by

$$
W L_{f}(G, H)=\sum_{(u, v) \in E(G)} d_{H}(f(u), f(v))=\sum_{e \in E(H)} E C_{f}(G, H(e))
$$

where $d_{H}(f(u), f(v))$ denotes the length of the path $P_{f}(u, v)$ in $H$. Then the wirelength of $G$ into $H$ is defined as,

$$
W L(G, H)=\min W L_{f}(G, H)
$$

where the minimum is taken over all the embeddings.

Definition 2.4. $[14,16]$ A circulant undirected graph, denoted by $G(n ; \pm S)$ where $S \subseteq\left\{1,2, \cdots,\left[\frac{n}{2}\right]\right\}, n \geq 3$ is defined as a graph consisting of the vertex set $V=\{0,1, \cdots, n$ $-1\}$ and the edge set $E=\{(i, j):|j-i| \equiv s(\bmod n), s \in S\}$. See Figure 1 .

Definition 2.5. [8] A wheel graph $W_{n}$ of order $\mathrm{n}$ is a graph that contains an outer cycle of order $n-1$, and for which every vertex in the cycle is connected to one other vertex (which is known as the hub). The edges of a wheel which include the hub are called spokes. See Figure 2(a). This plays an important role in the circuit layout and interconnection network designs.

Definition 2.6. [8] A gear graph, denoted by $G_{n}$ is a graph obtained by inserting an extra vertex between each pair of adjacent vertices on the perimeter of a wheel graph $W_{n}$. Thus, $G_{n}$ has $2 n+1$ vertices and $3 n$ edges. Gear graphs are also known as cogwheels and bipartite wheels. See Figure 2(b).

Definition 2.7. [8] The helm graph $H_{n}$ is the graph obtained from an wheel graph $W_{n}$, by adjoining a pendent edge at each node of the cycle. See Figure 2(c).

Lemma 2.8. (Congestion lemma) [11] Let $G$ be an $r$-regular graph and $f$ be an embedding of $G$ into $H$. Let $S$ be an edge cut of $H$ such that the removal of edges of $S$ leaves $H$ into 2 components $H_{1}$ and $H_{2}$ and let $G_{1}=f^{-1}\left(H_{1}\right)$ and $G_{2}=f^{-1}\left(H_{2}\right)$. Also $S$ satisfies the following conditions:

(i) For every edge $(a, b) \in G_{i}, i=1,2, P_{f}(f(a), f(b))$ has no edges in $S$. 
Jasintha Quadras and S. Sarah Surya

(ii) For every edge $(a, b)$ in $G$ with $a \in G_{1}$ and $b \in G_{2}, P_{f}(f(a), f(b))$ has exactly one edge in $S$.

(iii) $G_{1}$ is a maximum subgraph on $k$ vertices where $k=\left|V\left(G_{1}\right)\right|$.

Then $E C_{f}(S)$ is minimum and $E C_{f}(S)=r k-2\left|E\left(G_{1}\right)\right|$.

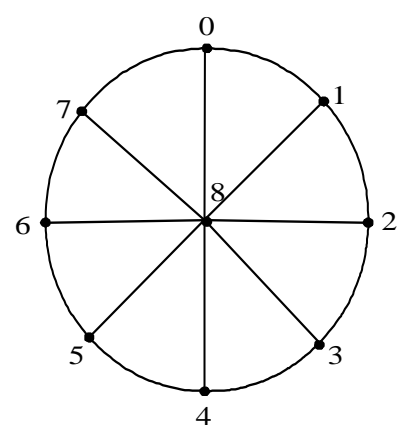

(a)

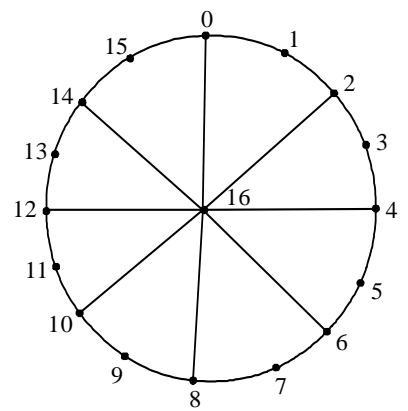

(b)

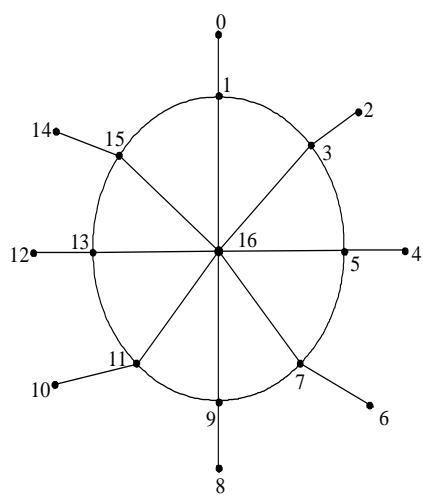

(c)

Figure 2:(a) Wheel graph (b) Gear graph (c) Helm graph

Lemma 2.9. (Partition lemma) [11] Let $f: G \rightarrow H$ be an embedding. Let $\left\{S_{1}, S_{2}, \ldots, S_{p}\right\}$ be a partition of $E(H)$ such that each $S_{i}$ is an edge cut of $H$. Then,

$$
W L_{f}(G, H)=\sum_{i=1}^{p} E C_{f}\left(S_{i}\right) .
$$

Lemma 2.10. ( $k$-Partition Lemma) [11] Let $f: G \rightarrow H$ be an embedding. Let $[k E(H)]$ denote a collection of edges of $H$ with each edge in $H$ repeated exactly $k$ times. Let $\left\{S_{1}, S_{2}, \ldots, S_{p}\right\}$ be a partition of $[k E(H)]$ such that each $S_{i}$ is an edge cut of $H$. Then

\section{Embedding algorithms}

$$
W L_{f}(G, H)=\frac{1}{k} \sum_{i=1}^{p} E C_{f}\left(S_{i}\right) .
$$

Theorem 3.1. [14] The number of edges in a maximum subgraph on $k$ vertices of $G(n ; \pm S), S=\{1,2, \cdots, j\}, 1 \leq j \leq\lfloor n / 2\rfloor, n \geq 3$ is given by,

$$
\xi=\left\{\begin{array}{c}
\frac{k(k-1)}{2}, k \leq j+1 \\
k j-\frac{j(j+1)}{2}, j+1<k \leq n-j \\
\left(\frac{1}{2}\right)\{(n-k) 2+(4 j+1) k-(2 j+1) n\}, n-j<k \leq n
\end{array}\right.
$$

Theorem 3.2. [14] A set of $k$ consecutive vertices of $G(n ; \pm 1), 1 \leq k \leq n$ induces a maximum subgraph of $G(n ; \pm S)$ where $S=\{1,2, \cdots, j\}, 1 \leq j \leq\lfloor n / 2\rfloor, n \geq 3$.

\section{Embedding Algorithm A}

Input: A circulant network, $G(2 n+1 ;\{1,2, \cdots, j\}), 1 \leq j \leq\lfloor n / 2\rfloor, n \geq 3$ and a gear graph, $G_{2 n+1}$ 
Wirelength of Circulant Networks into Wheel Related Graphs

Output: An embedding $f$ of $G(2 n+1 ;\{1,2, \cdots, j\})$ into $G_{2 n+1}$ given by $f(x)=x$ with minimum wirelength.

Algorithm: Label the consecutive vertices of $G(2 n+1 ;\{1,2, \cdots, j\}), 1 \leq j \leq\lfloor n / 2\rfloor, n \geq 3$ as 0 , $1, \ldots, 2 n$ in the clockwise sense. Label the outer cycle vertices of $G_{2 n+1}$ as $0,1, \ldots, 2 n-1$ and label the hub vertex as $2 n$.

\section{Proof of correctness of Algorithm A}

Let $S_{i}=\{(2 i-2,2 i-1),(2 i+1,2 i+2),(2 n, 2 i)\}, 1 \leq i \leq n$ where the labels are taken mod $(2 n)$ except the label of the hub vertex be the edge cuts of the given graph. The edge sets namely $\{(2 i-2,2 i-1),(2 i+1,2 i+2),(2 n, 2 i)\}, 1 \leq i \leq n$ constitutes all the edges of $\mathrm{G}_{2 n+1}$. Thus $\left\{S_{i},: 1 \leq i \leq n\right\}$ is a partition of $\left[E\left(G_{2 n+1}\right)\right]$. Thus $\left\{\mathrm{S}_{1}, \mathrm{~S}_{2}, \cdots, \mathrm{S}_{n}\right\}$ is a partition of $\left[E\left(G_{2 n+1}\right)\right]$. See Figure 3(a). For each $i, E\left(\mathrm{G}_{2 \mathrm{n}+1} \backslash \mathrm{S}_{\mathrm{i}}\right)$ has two components $H_{i 1}$ and $H_{i 2}$. Without loss of generality, let $H_{i 1}=\{(i, i+1),(i+1, i+2)\}$. Let $G_{i 1}=f^{-1}\left(H_{i 1}\right)$ and $G_{i 2}=f$ ${ }^{-1}\left(H_{i 2}\right)$. Then $G_{i l}$ induces an edge of $G$ which by Theorem 3.2 is an optimal set. Thus each $S_{i}$ satisfies conditions (i), (ii) and (iii) of the Congestion Lemma. Therefore, $E C_{f}\left(S_{i}\right)$ is minimum. The Partition Lemma implies that the wirelength is minimum.

The proof of the following theorem is an easy consequence of Embedding Algorithm A.

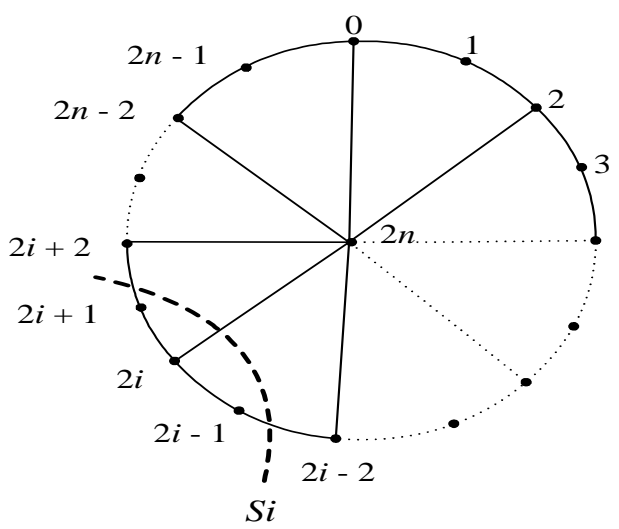

Figure 3(a): Edge cuts of gear graph

Theorem 3.3. The exact wirelength of $G(2 n+1 ;\{1,2, \cdots, j\})$ into $G_{2 n+1}, n \geq 3$, is given by $W L\left(G(2 n+1 ;\{1,2, \cdots, j\}), G_{2 n+1}\right)=2(3 n j-\xi)$.

\section{Embedding Algorithm B}

Input: A circulant network, $G(2 n+1 ;\{1,2, \cdots, j\}), 1 \leq j \leq\lfloor n / 2\rfloor, n \geq 3$ and a helm graph, $H_{2 n+1}$.

Output: An embedding $f$ of $G(2 n+1 ;\{1,2, \cdots \cdot j\})$ into $H_{2 n+1}$ given by $f(x)=x$ with minimum wirelength.

Algorithm: Label the consecutive vertices of $G(2 n+1 ;\{1,2, \cdots, j\}), 1 \leq j \leq\lfloor n / 2], n \geq 3$ as 0 , $1, \ldots, 2 n$ in the clockwise sense. Label the vertices of $H_{2 n+1}$ as follows:

(i) Label the pendant vertices consecutively as $0,2, \ldots, 2 n-2$ in the clockwise sense.

(ii) Label the vertices on the cycle consecutively as $1,3, \ldots, 2 n-1$ in the clockwise sense. 


\section{Jasintha Quadras and S. Sarah Surya}

(iii) Label the hub vertex as $2 n$.

\section{Proof of correctness of Algorithm B}

Let $S_{i}=\{(2 i-2,2 i-1)\}, 1 \leq i \leq n$, and let $S_{i}^{\prime}=\{(2 i-1,2 i+1),(2 i+3,2 i+5),(2 n, 2 i+1),(2 n, 2 i$ $+3)\}, 1 \leq i \leq n$, where the labels are taken $\bmod (2 n)$, except the label of the hub vertex be the edge cuts of the given graph. The edge set $\left\{S_{i},(2 i-1,2 i+1),(2 n, 2 i+1): 1 \leq i \leq n\right\}$ constitutes all the edges of $H_{2 n+1}$ exactly once. Similarly, the edge set $\left\{S_{i},(2 i+3,2 i+5)\right.$, $(2 n, 2 i+3): 1 \leq i \leq n\}$ constitutes all the edges of $H_{2 n+1}$ exactly once. Thus $\left\{S_{i}, S_{i}^{\prime}: 1 \leq i \leq\right.$ $n\}$ is a partition of [ $\left.2 E\left(H_{2 n+1}\right)\right]$. See Figure 3(b). For each $i, 1 \leq i \leq n, E\left(H_{2 n+1} \backslash S_{i}\right)$ has two components $H_{i 1}$ and $H_{i 2}$. Without loss of generality, let $H_{i 1}=\{(2 i-2)\}$. Let $G_{i 1}=f^{-1}\left(H_{i 1}\right)$ and $G_{i 2}=f^{-1}\left(H_{i 2}\right)$. Then $G_{i 1}$ induces an edge of $G$ which by Theorem 3.2 is an optimal set. Thus each $S_{i}$ satisfies conditions (i), (ii) and (iii) of the Congestion Lemma. Therefore, $E C_{f}\left(S_{i}\right)$ is minimum. Similarly, for each $i, 1 \leq i \leq n, E\left(H_{2 n+1} l S_{i}^{\prime}\right)$ has two components $H_{i 1}^{\prime}$ and $H_{i 2}^{\prime}$. Without loss of generality, let $H_{i 1}^{\prime}=\{(2 i, 2 i+1),(2 i+2,2 i+$ $3)\}$ for $1 \leq i \leq n$. Let $G_{i I}^{\prime}=f^{-1}\left(H_{i 1}^{\prime}\right)$ and $G_{i 2}^{\prime}=f^{-1}\left(H_{i 2}^{\prime}\right)$. Then $G_{i 1}^{\prime}$ induces an edge of $G$ which by Theorem 3.2 is an optimal set. Thus each $S_{i}^{\prime}$ satisfies conditions (i), (ii) and (iii) of the congestion lemma. Therefore, $E C_{f}\left(S_{i}^{\prime}\right)$ is minimum. The 2-Partition Lemma implies that the wirelength is minimum.

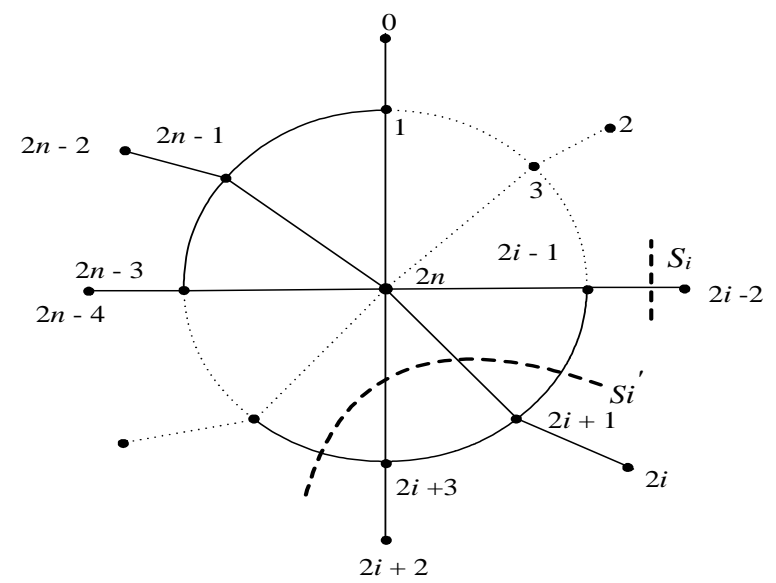

Figure 3(b): Edge cuts of helm graph

The proof of the following theorem is an easy consequence of Embedding Algorithm B.

Theorem 3.4.The exact wirelength of $G(2 n+1 ;\{1,2, \cdots, j\})$ into $H_{2 n+1}, n \geq 3$, is given by $W L\left(G(2 n+1 ;\{1,2, \cdots, j\}), H_{2 n+1}\right)=6 j n-\xi$.

\section{Conclusion}

In this paper, we have produced the exact wirelength of circulant network on certain wheel related graphs namely, gear and helm graphs.

\section{REFERENCES}

1. S.L.Bezrukov, Embedding complete trees into the hypercube, Discrete Applied Mathematics, 110(2-3) (2001) 101 - 119. 
Wirelength of Circulant Networks into Wheel Related Graphs

2. S.L.Bezrukov, J.D.Chavez, L.H.Harper, M.Rottger and U.P.Schroeder, Embedding of hypercubes into grids, MFCS, Electronic Edition Springer, Lecture Notes in Computer Science 1450, 693-701, 1998.

3. S.L.Bezrukov, B.Monien, W.Unger and G.Wechsung, Embedding ladders and caterpillars into the hypercube, Discrete Applied Mathematics, 83 (1998) 21-29.

4. T. Dvo'r'ak, Dense sets and embedding binary trees into hypercubes, Discrete Applied Mathematics, 155(4) (2007) 506 - 514.

5. J.-F.Fang and K.-C.Lai, Embedding the incomplete hypercube in books, Information Sciences, 96 (2005) 1-6.

6. M.R.Garey and D.S.Johnson, Computers and Intractability, A Guide to the Theory of NP-Completeness, Freeman, San Francisco, (1979).

7. A.K.Gupta, D.Nelson and H.Wang, Efficient embeddings of ternary trees into hypercubes, Journal of Parallel and Distributed Computing, 63(6) (2003) 619 - 629.

8. I.I.Javaid and S.Shokat, On the partition dimension of some wheel related graphs, Journal of Prime Research in Mathematics, 4 (2008) 154-164.

9. P.Manuel, Minimum average congestion of enhanced and augmented bypercube into complete binary tree, Discrete Applied Mathematics, 159(5) (2010) 360 - 366.

10. P.Manuel, M.Arockiaraj, I.Rajasingh and B.Rajan, Embedding hypercubes into cylinders, snakes and caterpillars for minimizing wirelength, Discrete Applied Mathematics, 159(17) (2011) 2109 - 2116.

11. P.Manuel, I.Rajasingh, B.Rajan and H.Mercy, Exact wirelength of hypercube on a grid, Discrete Applied Mathematics, 157(7) (2009) 1486 - 1495.

12. J.Opatrny and D.Sotteau, Embeddings of complete binary trees into grids and extended grids with total vertex congestion 1, Discrete Applied Mathematics, 98 (2000) 237-254.

13. M.Pal, Intersection graphs: An introduction, Annals of Pure and Applied Mathematics, 4(1) (2013) 43-91.

14. I.Rajasingh, P.Manuel, M.Arockiaraj and B.Rajan, Embeddings of circulant networks, Journal of Combinatorial Optimization, 11(3) (2011) 360 - 366.

15. I.Rajasingh, B.Rajan and R.S.Rajan, On embedding of m-sequential k-ary trees into hypercubes, Applied Mathematics, 1(6) (2010) 499 - 503.

16. J.M.Xu, Topological Structure and Analysis of Interconnection Networks, Kluwer Academic Publishers, (2001). 